\title{
The use of ultrasonography for the confirmation of pulled elbow treatment
}

\author{
Farhad Heydari', Shiva Samsam Shariat ${ }^{2 *}$, Saeed Majidinejad', Babak Masoumi' \\ 'Emergency Medicine Research Center, Alzahra Hospital, Department of Emergency Medicine, Isfahan University of Medical \\ Sciences, Isfahan, Iran \\ ${ }^{2}$ Emergency Medicine Department, Alzahra Hospital, Medical University of Isfahan, Isfahan, Iran
}

\author{
Received: 9 June 2017 \\ Accepted: 24 August 2017 \\ Published online: 10 September 2017 \\ *Corresponding author: Shiva \\ Samsam Shariat, \\ Tel: +989133112951, Email: \\ samsamshariat84@yahoo.com \\ Competing interests: None \\ Funding information: None
}

Citation: Heydari F, Samsam Shariat S, Majidinejad S, Masoumi B. The use of ultrasonography for the confirmation of pulled elbow treatment. Journal of Emergency Practice and Trauma 2018; 4(1): 24-28. doi: 10.15171/jept.2017.24.

\begin{abstract}
Objective: The aim of this study was to use ultrasonography for the diagnosis and confirmation of Pulled Elbow treatment.

Methods: This descriptive cross-sectional study initiated in 2014 and continued until 2015. We used simple sampling method and recruited 60 samples among patients aged 4 months to 6 years. The apparatus used in this study was an ultrasonogram with transducer $12 \mathrm{MHz}$ probe. Ultrasound evaluation of both hands was undertaken and after reduction, the physical examination was performed to confirm the diagnosis made by ultrasonography. Then, the results were recorded by a physician in a checklist and entered into SPSS software (version 20) for further analysis.

Results: In this study, 60 children with pulled elbow injuries were studied. Of these, 27 patients (45\%) were girls (female) and 33 (55\%) were boys (male). This indicates the higher incidence of injury among males than females. The highest incidence of pulled elbow injury was observed in children aged $3(15 \%)$. The accuracy of ultrasonography method for the confirmation of treatment was reported to be $92 \%$.

Conclusion: This study aimed to confirm the considered therapeutic method based on the result of ultrasonography performed after the treatment. Accordingly, the sensitivity and specificity of ultrasonography in confirming the considered therapeutic method for the treatment of pulled elbow was obtained higher than $90 \%$.

Keywords: Ultrasonography, X-ray, Pulled elbow injury
\end{abstract}

\section{Introduction}

Pulled elbow occurs most often in children (1-3); particularly those younger than 5 years old (4). Formerly, it was thought that it only happens in young children; but injuries similar to pulled elbow, such as elbow, trigger or subluxation of the annular ligament are also seen in adults (5). Pulled elbow in children usually occurs when adults or taller persons suddenly pull the child's hand from the elbow when it is stretched or when the child pulls his/ her hand suddenly from the hand of adults. During this incidence, radial bone is pulled by the annular ligament, resulting in dislocation of radial head (partial dislocation). Sometimes, ligament is torn off slightly and it is trapped between the head of radial bone and capitellum $(1,3,6)$. Common symptoms of this injury is to hear a low sound of breaking, or the child suddenly breaks down in tears because of pain and refuses to use his/her arm. The arm is bent and wrapped without a trace of swelling and bruising. The pain is usually felt in the elbow; but sometimes it may be felt in the wrist or shoulder. The elbow is able to do flexion and extension movements; but twisting of the forearm is faced with resistance and causes pain in the elbow joint $(1,6)$. The incidence rate of pulled elbow is rare, but its prevalence in children 0 to 5 years in Scotland Aberdeen city has been reported annually as equal to $1.2 \%$. On the basis of annual statistics of Wales and England, the incidence rate of the pulled elbow has been reported as 50000 cases annually (6). This injury is more common in the left hand and among girls (7).

Based on the studies conducted by Brown in 2009, the prevalence of emergency examinations for pulled elbow among children aged 0 to 18 years was reported as 2.7 per 1000 cases (8). The diagnosis is usually performed based on the patient's history and clinical examination. $(1,2,4)$; In cases where the patient's history is not clear, X-ray is required. X-ray can distinguish the elbow fractures. It can also detect other abnormalities of osteochondritis. Sometimes children experience a complete release of pain during the process of radiography. This is because of turning the child's radial (forearm) outside by X-ray 
technician in order to get a correct picture of the elbow which inadvertently treats the abnormality. Elbow ultrasonography can also be helpful (4).

Ultrasonography can also provide clinical information useful in evaluating the wide range of pathological conditions affecting the synovial space and surfaces of joints, such as tendon insertion and the soft tissues of the elbow joint. Elbow ultrasonography is an examination dependent on the skill of the machine operator. In addition, the experience of machine operator, knowledge of surgery, and definition of disease for providing a reliable report should be taken into account as well. Elbow ultrasonography has privileges compared to X-ray, including its usefulness in terms of the price and time, as well as higher distance resolution capability. Thus, ultrasonography provides the possibility of performing examination of the patient in a comfortable position (9). Ultrasonography examination of the elbow joint allows the examiner to distinguish between cartilage from the bone and soft tissues and shows changes in anatomical relationships by different views and levels. Ultrasonography makes it easy to compare the pulled elbow with the normal contralateral elbow. Besides, it is useful for the detection of thickening and sometimes annular ligament tear, dislocations of radial head, as well as measuring the distance between radial head and capitellum when the forearm is rotated inward. Ultrasonography can be used as a tool to measure progress and result of recurrent dislocation of the bone during treatment (2).

In a normal ultrasonography image, annular ligament is seen as a high-resonance linear image. A normal image of annular ligament is not always available in all cases of pulled elbow, and supinator muscle originating from annular ligament is trapped within the radiohumeral joint; and a low-resonance image is displayed in the J-shape form. In the contralateral elbow, all cases displayed normal images. After recovery and healing, J-shaped and low-resonance image was disappeared in all cases and annular ligament was seen in its original location. Sensitivity, specificity, and accuracy of ultrasonography in the diagnosis of J-shaped low resonance imaging were $100 \%$ for three above-mentioned factors (10).

Pathema principles were considered for posterior to anterior pulled elbow, radial head dislocation, and placement of annular ligament inside the radiohumeral joint (10). Imaging of the pulled elbow, which has been reported in the literature, is limited to the indirect detection of abnormal findings based on the positional relationship between the bone and detection of annular ligament (11).

Kosuwon et all (12) was the first person who used ultrasonography for documenting and confirmation of pulled elbow. In his sonographic study on the outer surface of radiohumeral joint in the long axis, he reported that finding the distance between radial and capitellum in the injured elbow, when it is compared with the normal elbow, is of value for diagnosis (13). However, his findings were not on the basis of annular ligament pathema, and they were solely based on detecting secondary changes of the joint (10).

Formerly, in an attempt to distinguish between healthy annular ligament, and trapped and torn annular ligament, ultrasound techniques for evaluation of pulled elbow were focused on the relation between capitellum and radial head (14).

However, assessment of morphological details of annular ligament and supinator muscle originating from annular ligament, which is in close contact with the pulled elbow pathology is considered difficult with sonographic study of the exterior surface of the radiohumeral joint in the long axis. In contrast, Minagawa was the first person who reported 32 cases of pulled elbow in his sonographic study of the anterior surface of the radiohumeral joint in the long axis; where the muscle originating from annular ligament forms a J-shape with the annular ligament trapped in the radiohumeral joint. He termed this finding as the "J-sign", and reported them as specific findings of the pulled elbow ultrasonography (15).

J-sign was observed in all our cases, which is consistent with and confirms the findings mentioned by Minagawa in his report. Ultrasonography provides a confirmed diagnosis of pulled elbow which can predict the recovery time using dynamic images.

Therefore, the use of ultrasonography could be strongly effective in diagnosis and healing of pulled elbow, even in cases of pulled elbow with unknown causes, where even low sounds are not heard and healing time is uncertain (10).

Some terms like nursemaid's elbow and pulled elbow are used for this abnormality. However, when it is possible to determine the pathological anatomy of this abnormality by ultrasonography, the use of the term "entrapment of the annular ligament in children" is more appropriate. It is possible to obtain a visible image of pulled elbow by ultrasonography. J-sign is disappeared with recovery and healing. A satisfactory recovery can be confirmed by observing normal relationships between radial head, capitellum, and annular ligament which is similar to that of the contralateral elbow. Ultrasonography is both a diagnostic test and a test for documenting and confirming a satisfactory recovery (10).

Given the common use of ultrasonography, its lower costs, spending less time, as well as using no radiation, we decided to conduct a study in which ultrasonography is used for the confirmation of pulled elbow treatment.

\section{Methods}

This cross-sectional study began in 2014 and continued until 2015 when 60 samples were collected using simple sampling method. 
Inclusion criteria encompassed willingness of patients to participate in the study, patients suffering from Pulled elbow injury, and being in the age range of 4 months to 6 years. Those who did not agree to take part in the study were excluded.

The apparatus used in this study was an ultrasonogram with $12 \mathrm{MHz}$ transducer probe. The patients kept their hands in the extension, then ultrasonography was performed after reduction on the anterior surface of radiohumeral joint in the long axis. Ultrasonography was conducted on both hands of the patients. After reduction, the physical examination was performed and ultrasonography was conducted to confirm the treatment. To reduce test error, a physician was asked to do ultrasonography with one ultrasonogram. A J-shape view in radiohumeral joint was observed in unltrasonography of the pulled elbow.

Results were recorded by a physician in a checklist. Descriptive statistics and susceptibilities were used to describe the data using SPSS software version 20.0.

\section{Results}

In this study, 60 children with pulled elbow injuries were studied. Of these, 27 patients (45\%) were girls (female) and 33 (55\%) were boys (male), indicating the higher incidence rate of this injury in boys than in girls (Table 1). The average age of patients was 2 years and 7 months with a standard deviation of 1.178 (2.7 \pm 1.178$)$. The majority of patients (65\%) were in the age range of 1 year and 9 months to 3 years and 10 months, and the highest prevalence of pulled elbow injury was observed in children aged 3 (15\%). Of a total of 60 patients studied, 24 patients were suffering from right elbow injury and 36

Table 1. A summary of descriptive statistics of the studied independent variables ${ }^{\mathrm{a}}$

\begin{tabular}{llc}
\hline & & Incidence rate, No. (\%) \\
\hline \multirow{3}{*}{ Gender } & Male & $33(55 \%)$ \\
& Female & $27(45 \%)$ \\
& Total & 60 \\
\hline \multirow{3}{*}{ The involved elbow } & Right & $24(40 \%)$ \\
& Left & $36(60 \%)$ \\
& Total & 60 \\
\hline
\end{tabular}

${ }^{a}$ Age (years), mean \pm standard deviation: $2.7 \pm 1.178$. patients were suffering from left elbow injury. Therefore, it can be said that the left elbow injury is most common in children (60\%).

This study aimed to perform ultrasonography to confirm the treatment of pulled elbow. Accordingly, from a total of 60 patients, $49(81.6 \%)$ participants had a normal ultrasonographic examination, and 11 (18.4\%) participants did not have normal ultrasonography. From the 11 patients who had abnormal ultra sound, 5 patients were referred to orthopedic surgeon based on more checking and observing a fracture in the elbow area. In 6 patients due to obesity or excessive weight loss, ultrasound was not successful and reliable, thus they were observed in the emergency department. After relaxation, among the six children, four of them were re-examining discharged. As agitation continued in 2 of the 6 children, and owing to unablility to perform the correct examination, X-ray was performed and they were discharged.

True positive patients (the number of patients who have complete treatment and normal ultrasonography) were 49 and false positive patients (the number of patients with complete treatment and abnormal ultrasonography) were 6 in number (Table 2).

Positive predictive value (PPV) is the percentage of persons that have positive test (normal ultrasound of radiohumeral joint) and the complete treatment is 1 . Negative predictive value (NPV) is the percentage of patients that have negative test (they do not have normal ultrasound of radiohumeral joint) and the orthopedic pathology is 0.45 (Table 2).

In our study the sensitivity and specificity of ultrasonography in confirming the treatment of pulled elbow was $89.1 \%$ and $100 \%$ and The accuracy of ultrasonography method in confirming the treatment of pulled elbow was reported $92 \%$.

\section{Discussion}

In this study according to our results, the sensitivity and specificity of ultrasonography in confirming the considered therapeutic method for the treatment of pulled elbow was obtained higher than 90\%. Therefore, ultrasonography has different advantages such as high resolution and speed for the confirmation of pulled elbow treatment. Our findings are consistent with the study

Table 2. Frequency of ultrasonography results based on patient's treatment (medical) condition ${ }^{\text {a }}$

\begin{tabular}{llrr}
\hline & & \multicolumn{2}{c}{ The patient's condition } \\
\cline { 3 - 4 } & & $\begin{array}{c}\text { Treated (discharged), } \\
\text { No. (\%) }\end{array}$ & $\begin{array}{c}\text { Untreated (Hospitalization and } \\
\text { referral to an orthopedist), No. (\%) }\end{array}$ \\
\hline $\begin{array}{l}\text { Ultrasonography } \\
\text { results }\end{array}$ & Normal (reduction) & $49(89.1)$ & $0(0)$ \\
& Abnormal (no reduction, no ligament) & $6(89.1)$ & $5(100)$ \\
5
\end{tabular}

a $P P V=1, N P V=0.45$. 
conducted by Lee et al. They reported a case of an arm wrestler with an MCL injury diagnosed using dynamic ultrasonography (16). In their investigation, a 58-yearold man injured his left elbow while arm wrestling. They compared differences of ultrasonography and dynamic ultrasound (US) in the assessment and evaluation of elbow MCL injury. Initially they evaluated elbow injuries with ultrasonography and Dynamic US. Their patient presented with an MCL tear in the left elbow and at the 3-year follow-up ultrasound examination, an MCL tear in the right elbow was also suspected. Resting US did not show an MCL tear, whereas the tear was evident with a dynamic US study. They concluded that Dynamic US is indispensable in the evaluation of elbow injuries. Also, ultrasonography is a useful imaging modality to evaluate elbows with possible MCL injury, as it may show the torn component of the MCL. As a result of advanced technologies, US can offer dynamically focused higher resolution and real-time imaging of soft tissues. Therefore, advantages include its safety (nonionizing radiation), accessibility, speed, comfort, and cost-effectiveness. Finally, they emphasized that US is a highly useful imaging modality for the diagnosis of elbow MCL injury. Also, Dynamic US can be used to assess certain conditions such as muscle herniation, and is an indispensable technique in the evaluation of elbow injury. So, the result of this case report is in line with our study.

A study investigating 2331 cases of pulled elbow over the last 10 years by Irie et al is inconsistent with our study (17). In this study, all pediatric patients with a diagnosis of pulled elbow from January 2002 to December 2011 were studied according to sex, age, affected arm, recurrence rate, mechanism of injury and treatment outcomes. They found that the frequency of injury peaked for both boys and girls at 6 months and 2 years of age and also the left arm was more affected than the right. Previous studies of pulled elbow concluded that the injury typically occurs between the ages of 1 and 4 years with a peak incidence between 2 and 3 years $(18,19)$. However, the results of this study represented that it often occurs also in children who are less than 1 year old. Also, they found that the left arm is more commonly affected than the right. In addition, the frequency of injuries to the left arm tends to increase with age but the cases injured with other histories have a low percentage of injuries in this study. So, radiographs should be used before reduction when the history of the patients shows no case of pulling.

\section{Conclusion}

This study aimed to confirm the considered therapeutic method based on the result of ultrasonography performed after the treatment. Accordingly, the sensitivity and specificity of ultrasonography in confirming the considered therapeutic method for the treatment of pulled elbow was obtained higher than $90 \%$.

\section{Ethical issues}

This study was approved by Ethic Committee of Isfahan University of Medical Sciences.

\section{Authors' contributions}

noitubirtnoc pihsrohtua rof airetirc ruof dessap srohtua llA lanoitanretnI eht fo snoitadnemmocer no desab srotidE lanruoJ lacideM fo eettimmoC

\section{Acknowledgments}

The authors appreciate the insightful cooperation of Medical Sciences and the staffs of the emergency department of Al-Zahra hospital and Kashani hospital, Isfahan, Iran.

\section{References}

1. Krul M, Van der Wouden JC, Koes BW, Schellevis FG, Van Suijlekom-Smit LW. Nursemaid's elbow: Its diagnostic clues and preferred means of reduction. J Fam Pract 2010; 59(1): E5-7.

2. Kim MC, Eckhardt BP, Craig C, Kuhns LR. Ultrasonography of the annular ligament partial tear and recurrent "pulled elbow”. Pediatr Radiol 2004; 34(12): 999-1004. doi: 10.1007/ s00247-004-1284-7.

3. Krul M, van der Wouden JC, van Suijlekom-Smit LW, Koes BW. Manipulative interventions for reducing pulled elbow in young children. Cochrane Database Syst Rev 2012; 1: CD007759. doi: 10.1002/14651858.CD007759.pub3.

4. Sevencan A, Aygün Ü, İnan U, Ömeroğlu H. Pulled elbow in children: a case series including 66 patients. J Pediatr Orthop B 2015; 24(5): 385-8. doi: 10.1097/BPB.0000000000000182.

5. Kajiwara R, Sunagawa T, Ishida O, Ochi M. Irreducible pulled elbow in an adult: a case report. J Shoulder Elbow Surg 2007;16(1):e1-4. doi: 10.1016/j.jse.2006.03.007.

6. Jongschaap HC, Youngson GG, Beattie TF. The epidemiology of radial head subluxation ('pulled elbow') in the Aberdeen city area. Health Bull (Edinb) 1990; 48(2): 58-61.

7. Hagroo GA, Zaki HM, Choudhary MT, Hussain A. Pulled elbow-not the effect of hypermobility of joints. Injury 1995; 26(10): 687-90. doi: 10.1016/0020-1383(95)00133-6.

8. Brown D. Emergency department visits for nursemaid's elbow in the United States, 2005-2006. Orthop Nurs 2009; 28(4): 161-2. doi: 10.1097/NOR.0b013e3181ada779.

9. Martinoli C, Bianchi S, Giovagnorio F, Pugliese F. Ultrasound of the elbow. Skeletal Radiol 2001; 30(11): 60514. doi: 10.1007/s002560100410.

10. Dohi D. Confirmed specific ultrasonographic findings of pulled elbow. J Pediatr Orthop 2013; 33(8): 829-31. doi: 10.1097/BPO.0000000000000087.

11. Scapinelli R, Borgo A. Pulled elbow in fancy: diagnostic role of imaging. Radiol Med 2005; 110(5-6): 655-64.

12. Kosuwon W, Mahaisavariya B, Saengnipanthkul S, Laupattarakasem W, Jirawipoolwon P. Ultra-sonography of pulled elbow. J Bone Joint Surg Br1993; 75(3): 421-2.

13. Taha AM. The treatment of pulled elbow: a prospective randomized study. Arch Orthop Trauma Surg 2000; 120(56): 336-7.

14. Diab HS, Hamed MM, Allam Y. Obscure pathology of 
pulled elbow: dynamic high-resolution ultrasound-assisted classification. J Child Orthop 2010; 4(6): 539-43. doi: 10.1007/s11832-010-0298-y.

15. Minagawa H. Diagnostic and interventional musculoskeletal ultrasound. J Jpn Orthop Assoc 2012; 86(11): 1057-64.

16. Lee YS, Chou YH, Chiou HJ, Lai YC . Use of sonography in assessing elbow medial collateral ligament injury after arm wrestling . J Chin Med Assoc 2014; 77(3): 163-5. doi: 10.1016/j.jcma.2013.11.003.

17. Irie T, Sono T, Hayama Y, Matsumoto T, Matsushita M.
Investigation on 2331 cases of pulled elbow over the last 10 years. Pediatr Rep 2014; 6(2): 5090. doi: 10.4081/ pr.2014.5090.

18. Schunk JE. Radial head subluxation: epidemiology and treatment of 87 episodes. Ann Emerg Med 1990; 19(9): 1019-23.

19. Macias CG, Bothner J, Wiebe R. A comparison of supination/flexion to hyperpronation in the reduction of radial head subluxations. Pediatrics 1998; 102(1):e10. 